\title{
Review Article \\ EEG Derived Neuronal Dynamics during Meditation: Progress and Challenges
}

\author{
Chamandeep Kaur and Preeti Singh \\ Panjab University, Chandigarh 160036, India \\ Correspondence should be addressed to Chamandeep Kaur; ckchauhan.86@gmail.com
}

Received 22 September 2015; Revised 11 November 2015; Accepted 15 November 2015

Academic Editor: Guido Dietrich

Copyright (C) 2015 C. Kaur and P. Singh. This is an open access article distributed under the Creative Commons Attribution License, which permits unrestricted use, distribution, and reproduction in any medium, provided the original work is properly cited.

\begin{abstract}
Meditation advances positivity but how these behavioral and psychological changes are brought can be explained by understanding neurophysiological effects of meditation. In this paper, a broad spectrum of neural mechanics under a variety of meditation styles has been reviewed. The overall aim of this study is to review existing scientific studies and future challenges on meditation effects based on changing EEG brainwave patterns. Albeit the existing researches evidenced the hold for efficacy of meditation in relieving anxiety and depression and producing psychological well-being, more rigorous studies are required with better design, considering client variables like personality characteristics to avoid negative effects, randomized controlled trials, and large sample sizes. A bigger number of clinical trials that concentrate on the use of meditation are required. Also, the controversial subject of epileptiform EEG changes and other adverse effects during meditation has been raised.
\end{abstract}

\section{Introduction}

Meditation is a broad variety of practices. Meditation includes relaxation supporting techniques and various movements to achieve regulation and nurturing of well-being. It is defined as the natural process of manipulating one's state of mind and self-regulating attention level intentionally, although it is lacking a clear operational definition $[1,2]$. Experienced meditators show stronger regulated features. Analysis of various meditation states has been done to explore difficult neural processes. The large and complex neuronal structures turn to more relaxed structures during meditation [3]. So meditation practitioners show distinct EEG recording patterns. In the beginning of each meditation style, Lutz et al. have categorized meditation as Focused Attention (FA) and open monitoring (OM). Travis represented another category as automatic self-transcending according to brain pattern based EEG bands. These categories define a number of meditation practices $[4,5]$. Minimum efforts are required to reach the state of FA and a steady focus is achieved which is evident from less activation in the amygdala [1].

Physiological and Neural Correlates of Meditation Therapy. Meditation practices can change the way of thinking.
Metacognitive reasoning could be made by people to predict the discrepancy between what should be thought and what is being thought. In [6], various neurophysiologic changes on cognition, hormonal, and autonomic systems have been theorized while meditating. Evidences reveal the positive effects of meditation as it increases the level of monoamines, parasympathetic activity, and gray matter density of brain regions (reflecting emotion regulation) and reduces oxidative effects. These effects result in reversal of stress mediated depression. Further research needs to be done in exploring these changes with large samples in consideration. The spectral power and coherence of EEG define delta, theta, and alpha frequency bands to characterize different meditation states.

Numerous researches have been carried out to scientifically explore the principle brain neurological changes during meditation. But neuroelectrophysiology of meditation based states is still an open question. A very small number of clinical applications of meditation have been identified and those too are lacking in control group and concurrent antidepressant medication group along with shorter follow-up period $[6,7]$. Albeit some progress has been made in theorizing the neurophysiological effects under meditation [4, 8-10], evoked 
potential, and event related potential research in meditation [11], still rationale to quantitatively represent the neural effects is not clear.

This review is a noteworthy contribution to review existing scientific studies and future challenges on meditation effects based on changing EEG brainwave patterns. A debate on the EEG changes during meditation, controversial adverse effects of meditation, and signal processing challenges with future direction has been given below.

\section{EEG during Meditation}

A detailed quantitative analysis of neural effects under the effect of various meditation states has been discussed below. Other studies on the EEG effects of meditation could be found in Table 1.

2.1. Buddhist Meditation. Based on open monitoring, Buddhist meditators are characterized by high amplitude gamma oscillations [1]. A study about the impact of Buddhist meditation on emotional processing was presented [12]. There was no difference in psychological testing in experimental and control group (CG). Also, randomized design should be incorporated in the study design.

2.1.1. Zen Meditation. Zen meditation, a type of Buddhist meditation, is characterized by increase in slow alpha power, reflecting more internalized attention, and fast theta power, reflecting enhanced mindfulness in frontal area $[9,13]$. Longterm practitioners show alphal rise in frontal region and beta rise over occipital regions [14].

Investigations on FmTheta. During a mental effort, EEG shows a decrease in alpha and this strength goes on decreasing as the task becomes more tedious. However, frontal midline theta (FmTheta) increases during mental task. FmTheta involves attention levels that appear during mental task and meditation. It is an indicator of more demonstrative, more active, and less phobic activities [15]. It is defined on EEG as distinctive theta activity in frontal midline area. A study was performed on theta signal during concentrative task (meditation) and consecutive mental task [16]. It refers to attention maintenance during mental effort. Enhanced theta and constant occipital alpha were observed between FmTheta conditions and control. Further investigations on FmTheta can add to the underlying mechanisms of mind and body.

2.1.2. CHAN Meditation. Researchers correlated occipital alpha wave rise during eye closed relaxation and frontal alpha wave (representing mindfulness) during CHAN meditation. A method explored spatial temporal properties of different alpha maps using microstate analysis in [17]. It reflected more inward attention than the CG. Another study showed that better cortical interactions are possessed during CHAN meditation and Chakra focusing practice than the CG [18]. In this study, interactions among various brain networks under alpha oscillations have been explored because of Continuous
Time Wavelet Transform. This nonlinear independence analysis identified the dominant alpha epochs in right and left temporal regions.

2.1.3. Mindfulness Meditation (MM). Meditators have reduced physiological arousal and distraction from vague thoughts. It can be used for treating ADHD [19]. MM can induce neuroplasticity from earlier stages in self-referential processing and increased attention to internal and external stimulus. Self-referential processing is related to DMN (Default Mode Network) [20-24].

This study theorized various state and trait effects of MM on self-referential processing. Cahn et al. have measured the effect of Vipassana in terms of decreased delta and relative increase in theta over the frontal regions, though other usual changes over alpha, beta, and theta bands have not been observed. The main limitation of the study was unspecific effect of meditation practice on different frequencies, which should be well understood [24].

Ahani et al. [25] advanced the meditation research on MM. They supported the evidence of alpha and theta rise by observing the spectral analysis of recorded EEG. Although this randomized controlled trial was analyzed using the CG, the study was limited to novice meditators only [25]. Other MM techniques of IBMT (Integrative Body-Mind Training) and TBRT (Triarchic Body Pathway Relaxation) have been explored but still very little information is available and large scale considerations of these techniques are required for expert meditators $[26,27]$.

2.2. Transcending Meditation (TM). Various physiological and neural effects in long-term Jacobson's Progressive Relaxation (PR) meditators (a classical method of relaxation), TM meditators, and group of beginners in $\mathrm{PR}$ were recorded [28]. Rare theta activity $(5-7 \mathrm{~Hz})$ was observed in all the three. Then, a detailed research on TM explained its effects that could be used for various clinical applications [29]. Comparing TM-Sidhi with TM program revealed higher frontal alphal and betal amplitudes and no change in coherence. Higher alphal and betal reflect automatic selftranscending and open monitoring, respectively. This analysis was conducted for frontal and parietal areas. For temporal amplitude averages, no significant changes were observed [30]. Limitation was no CG. It has also been made evident that TM could be used for stress reduction and improving the symptoms of ADHD (Attention Deficit Hyperactivity Disorder) [31,32]. In a study, individual and group meditation effects were analyzed [33]. During individual meditation with closed eyes, strong delta coherence and theta coherence and no alpha coherence were observed. Distinct changes in alpha and theta coherence during group meditation show more relaxation reflected by subjects and less delta activity.

In another study, effects of TM on EEG alpha phase synchrony have been studied [34]. An increase in synchrony was observed around frontal and occipitoparietal lobes by a time domain method. It accounts for use of this style of meditation for better concentrated neural processes. Event related spectral perturbation (ERSP) analysis of EEG signal 


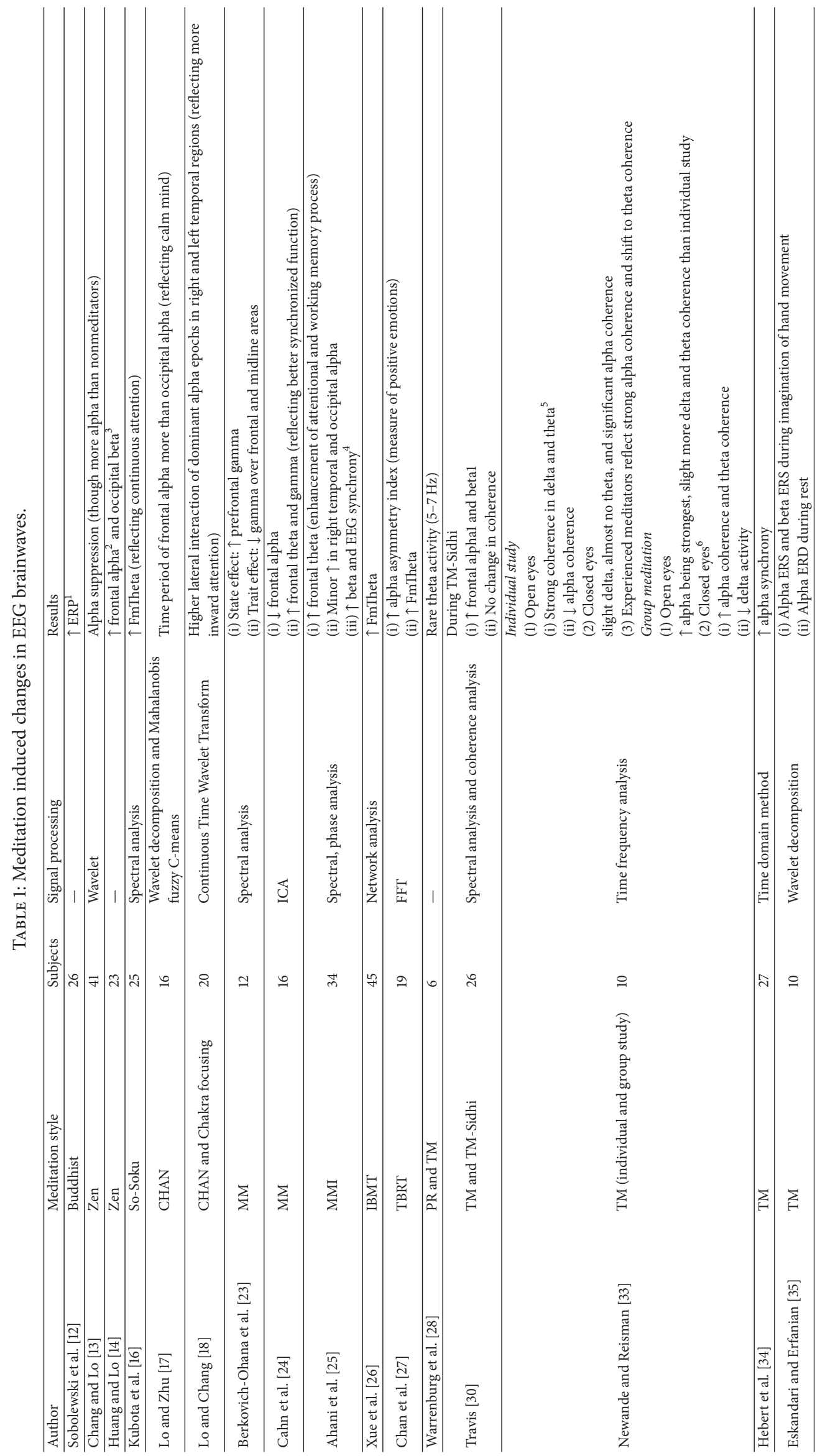




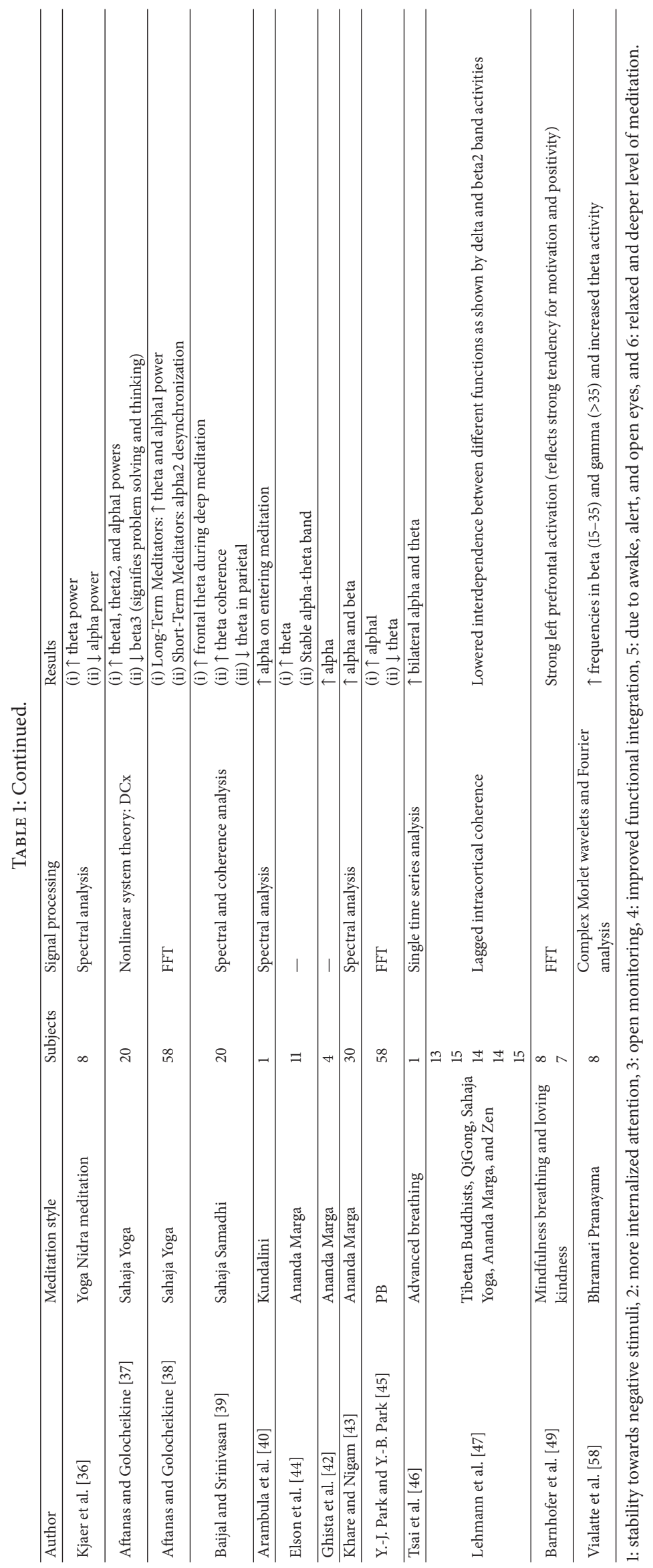


during TM practice has been presented for analyzing mind controllability of brain-computer interaction systems [35]. No CG was used for analysis.

2.3. Yoga Meditation. Evidences showed heightened theta EEG activity during Yoga Nidra and Sahaja Yoga but more control group designed study is needed [36, 37]. During Sahaja Yoga, LTM (Long-Term Meditators) showed higher theta and alphal power and STM (Short-Term Meditators) showed alpha2 desynchronization [38]. EEG mechanisms during Sahaja Samadhi (concentrative) meditation reflect increase in theta power and theta coherence in frontal area during deep meditation [39]. Again comparison with CG is required.

EEG pattern of a Kundalini Yoga master and Ananda Marga have been theorized as reflecting more alpha and theta band [40-44]. Still very few studies have been conducted for Yoga meditation considering the proficiency levels.

2.4. Other Meditation Practices. Paced Breathing (PB), which is a type of Su-Soku meditation, is a method of voluntary breathing [45]. It causes change in EEG parameters (increase in low frequency and high frequency alpha power and decrease in theta power). $\mathrm{PB}$ is advantageous compared to other meditative states [45].

An oscillatory mechanism of EEG during advanced breathing meditation, a new area to explore, has been studied in [46]. This finding supported the fact that the internalized attention is continuously enhanced and is noteworthy as reported by rise in theta activities, while relaxation is prominent only after deep phase of meditation as reported by changes in alpha activities [46].

2.5. Other Combined Studies. It has been revealed in a study that electric function connectivity differs in five traditions of meditation [47]. These are Tibetan Buddhists (TB), Qigong, Sahaja Yoga (SY), Ananda Marga Yoga (AY), and Zen [47]. The results have been taken for delta and beta2 bands because all five meditations showed significant changes in these two bands. In delta band of TB group, moving out of meditation showed left to right posterior connection while it showed anterior left to right posterior connectivity in AY group [47]. Such types of guesses evidently become impossible, for example, Qigong, since it includes a large number of connections. This research shows the common features in five traditions of meditation but with no CG and it did not explore the difference. Analysis of principal functional connectivities during delta band reflected that interdependence between different functions is lowered with practicing meditation irrespective of meditation style and inhibitory and excitatory (delta and beta 2 band activities) brain region connectivities show this reduction $[47,48]$.

In another study, state effects of two meditation styles, mindfulness breathing and loving kindness (or metta) meditation, have been investigated [49]. Effects on prefrontal alpha asymmetry have been discussed. Subjects low in brooding showed response to loving kindness meditation while the opposite was observed for subjects with high brooding.
Comparisons to rest group showed useful state effects of both the styles of meditation. It accounts for their clinical use for previously depressed patients, although various limitations like unspecific factors, small sample size, and novice subjects were observed in the study [49].

\section{Controversial Studies}

It has also been reported that meditation has an adverse effect of predisposing to epileptogenesis panic attacks, overexcited central nervous system, paradoxical rise in anxiety, becoming more hypercritical, disorientation, and high BP [10]. Regarding the use of meditation for high BP, more randomized clinical trials are required that could provide certain results [50]. Some positive effects might become negative if overexpressed in those with individual constitutional neurophysiological properties [EEG guided med.]. Deep meditation gives rise to high frequency gamma band bursts [51]. The patients with general epilepsy show increased gamma band activity especially $30-50 \mathrm{~Hz}$ in resting interictal EEG [52, 53]. Epileptiform EEG changes have been theorized in TM meditators. So some studies accounted for meditation resulting in epilepsy but some have rejected such claims [5157]. Meditation predisposing to epilepsy is a controversial subject that requires scientific study.

During Bhramari Pranayama (BhPr), paroxysmal gamma (PGW) has been observed [58]. Using complex Morlet wavelets and Fourier analysis, features were extracted as high frequencies in beta (15-35) and gamma (>35) and increased theta activity. BhPr can represent epileptic activity since higher frequency epilepsy also exhibits such spiky shape and activity in temporal lobe as seen in BhPr [58]. Another study has brought light to TM as aggravating or treating epilepsy [59].

\section{Signal Processing Challenges}

Signal processing can add to this field of study [60]. The scalp electrodes, which contain the brain activity in terms of electric potentials, give signals which can be processed using SP algorithms to measure different mental activities and to reveal cognitive tasks. The internal language of the mind can be understood using different EEG patterns from electromagnetic field activity [61, 62]. EEG includes signals that are associated with awareness, encouragement, and cognitive load and affecting state of load [63-65].

EEG data is characterized by delta, theta, alpha, beta, and gamma [66]. A detailed description of assigned EEG bands has been given in Table 2 .

A pronounced attempt to characterize EEG signatures for different types of meditation has been contributed by Travis and Shear [4]. They have not allocated rating but have valued the nature of meditation styles. A superior research in meditation is still required to categorize the EEG signatures corresponding to different meditation practices $[4,5]$.

Directly observing the nonlinear and nonstationary EEG raw data in time domain is a very tedious task [67]. So various linear and nonlinear signal processing techniques and 


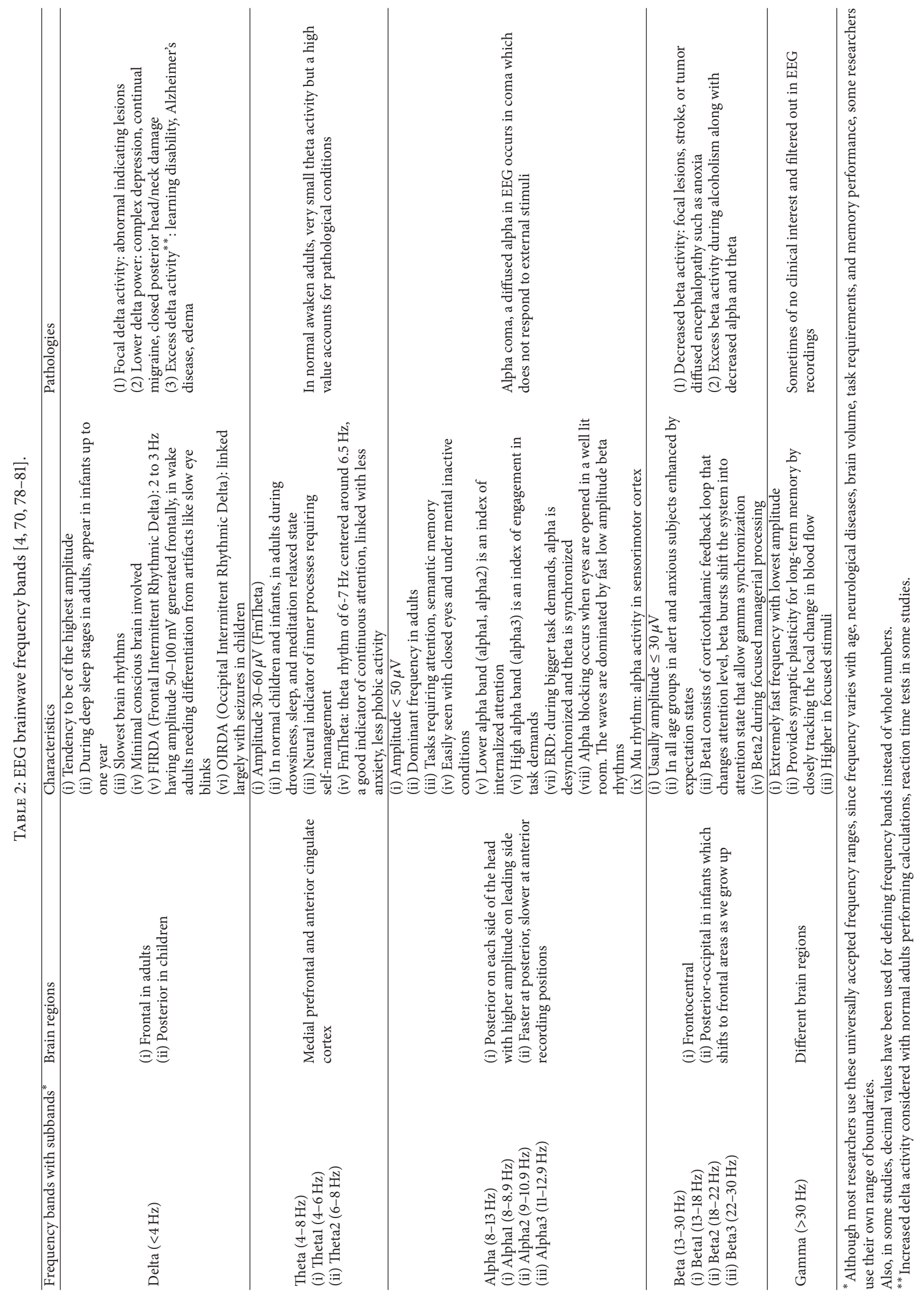


their correlation to physiology have been proposed. Feature extraction algorithms acquire the spectral information from the preprocessed raw signal.

Time frequency analysis is beneficial in clarifying rhythmic information in EEG signals. Coherence techniques can also be used. Spectral covariance or coherence involves measurement of phase regularity between signal pairs in each frequency band. Higher alpha-theta coherence has been identified as meditation capability trait which appears intra- and interhemispherical in meditation [68]. As coherence cannot separate amplitude information and phase information while relating two signals, it measures roundabout phase locking only. Synchrony technique is being used rather than having spectral or coherence analysis. It is quantification of degree of phase locking between different narrowband signals [69].

Linear methods used are ICA (Independent Component Analysis), CSP (Common Spatial Patterns), LD (Linear Discriminator), and linear prediction method [70]. In other words, a wide range of algorithms extract the information in EEG signals like algorithms based on Fast Fourier Transform, Hilbert-Huang Transform, Wavelet Transform, rule based expert systems, and numerous other algorithms to define the temporal extents [70-72]. A method based on PBFT (Period Based Frequency Tracker) has been proposed to keep track of rhythmic variations of alpha frequencies [73]. Though STFT (Short Time Fourier Transform) can analyze such spectral variations, this method proved better in terms of fairer frequency resolution [73]. Also, problem of asymmetrical periodicity of EEG has been solved reliably and efficiently.

The inherent inhomogeneous characteristics and multinature are dealt with using nonlinear signal processing techniques on the basis of various parameters, for example, CD (Correlation Dimension), LLE (Largest Lyapunov Exponent), and $\mathrm{H}$ (Hurst exponent) [70]. Four-channel analysis of EEG has been given for Compressed Spectral Array (CSA), the running fractal dimension, and running attractor dimension [74]. CSA yields interesting features. The running attractor is more efficient in analyzing neural dynamics. Multiscale fractal dimension (MSFD) technique is another area of research to explain the multiscale temporal patterns of EEG [75]. Nonlinear methods are better than time domain, frequency domain, and linear methods.

\section{Future Work and Challenges}

A crisp and consistent observation of a particular EEG component and its changing direction as either increasing or decreasing for different meditation practices is still required. Numerous researches have been carried out to scientifically explore the underlying brain neuroelectrical effects during meditation but still neurophysiological effect of meditation is an open question. Though it has been theorized that a typical EEG signature of meditation can be increase in theta, alpha band power, decrease in at least alpha frequency, and spread of alpha coherence across cortex, its realization needs further research [4]. Also finding a comparable nonmeditating group as a control group is very difficult [11]. The main limitation is absence of control group. Whether the changes in EEG signals classify the restrained states of consciousness is questionable, although a pronounced clarification has been contributed by Travis and Shear. Further investigations are required to quantitatively represent the activities during FmTheta and activities concerning DMN during different styles of meditation. For EEG during DMN, the proposed approaches can be cross power spectra techniques, partial coherence function computation techniques, and operational architectonics techniques. Further studies have been suggested to consider extra frequency ranges and cross-correlation between EEG signals [75]. Very few conclusions have been derived regarding the expertise level of Yoga meditation. Neurobiological study of various types of Buddhist, Yoga, and other meditations styles is still to be explored. Also, meditation predisposing to epileptic seizure as well as some other negative effects is a controversial subject that requires technical discussion.

Signal processing can add to this field of study. EEG is the best diagnostic tool to provide firm information about brain activities and temporal dynamics related to these activities within millisecond range but if analyzing technique is poor it could result in misinterpretation and may ignore certain neural correlates [67]. So it can be said that EEG is misjudged highly. More processing tools and engineering approaches are required to be investigated for exploring EEG information [76].

Also meditation effects on the brain activity measured by EEG could be contaminated by the electromuscular artifacts. EEG rhythms show 6 times less power in 25-30 Hz band and 100 times less $40-100 \mathrm{~Hz}$ power in paralyzed subjects [77]. So muscle contamination is an important issue in defining gamma EEG during meditation.

\section{Conclusion}

Nowadays, complementary therapies like meditation are starting to be used in clinical practices. Understanding neuroelectrical effects of meditation is an important area of research, especially considering the application of meditation techniques in clinical practice and therapy. Studies based on EEG brainwave signals can help medical practitioners to check the activity level of brain and based on the health state, different meditation practices can be applied to progress mental fitness. In this paper, a broad spectrum of neural mechanics under a variety of meditation styles has been reviewed. A detailed quantitative analysis of various meditation states like Zen, CHAN, mindfulness, TM, Vipassana, Kundalini, Yoga, and other meditation styles has been described by means of EEG bands and coherence. It has been concluded that increased independent brain processes are observed compared to task-free resting during meditation. More rigorous studies are required with better design, considering client variables like personality characteristics to avoid risks, randomized controlled trials, long-term effects, and large sample sizes. More rigorous clinical trials that concentrate on the use of meditation are also required. Investigations on further activities can add neuroanatomy to explore the mechanisms of mind and body. Signal processing aspects in the field of cognitive neuroplasticity have also been 
discussed. A superior research is still required to categorize the EEG signatures corresponding to different practices.

In summary, highly developed approaches to the research of meditation will scientifically explore the underlying brain functions which may be beneficial for social well-being. A large scale research has been done to explore the neurophysiologic effects of meditation but still much is to be done to explore this area as suggested by various studies.

\section{Conflict of Interests}

The authors declare that there is no conflict of interests regarding the publication of this review paper.

\section{Acknowledgment}

The authors would like to thank their faculty members who have given them great suggestions and support.

\section{References}

[1] R. J. Davidson and A. Lutz, "Buddha's brain: neuroplasticity and meditation," IEEE Signal Processing Magazine, vol. 25, no. 1, pp. 174-176, 2007.

[2] A. Lutz, H. A. Slagter, J. D. Dunne, and R. J. Davidson, "Attention regulation and monitoring in meditation," Trends in Cognitive Sciences, vol. 12, no. 4, pp. 163-169, 2008.

[3] N. Pradhan and N. D. Dutt, "An analysis of dimensional complexity of brain electrical activity during meditation," in Proceedings of the 1st Regional Conference IEEE Engineering in Medicine (EMBS '95) \& Biology Society and 14th Conference of the Biomedical Engineering Society of India (BMESI '95), pp. 1.92-1.93, February 1995.

[4] F. Travis and J. Shear, "Focused attention, open monitoring and automatic self-transcending: categories to organize meditations from Vedic, Buddhist and Chinese traditions," Consciousness and Cognition, vol. 19, no. 4, pp. 1110-1118, 2010.

[5] Z. Josipovic, "Duality and nonduality in meditation research," Consciousness and Cognition, vol. 19, no. 4, pp. 1119-1121, 2010.

[6] E. R. Kasala, L. N. Bodduluru, Y. Maneti, and R. Thipparaboina, "Effect of meditation on neurophysiological changes in stress mediated depression," Complementary Therapies in Clinical Practice, vol. 20, no. 1, pp. 74-80, 2014.

[7] N. T. Y. Leung, M. M. Lo, and T. M. C. Lee, "Potential therapeutic effects of meditation for treating affective dysregulation," Evidence-Based Complementary and Alternative Medicine, vol. 2014, Article ID 402718, 7 pages, 2014.

[8] B. R. Cahn and J. Polich, "Meditation states and traits: EEG, ERP, and neuroimaging studies," Psychological Bulletin, vol. 132, no. 2, pp. 180-211, 2006.

[9] T. Takahashi, T. Murata, T. Hamada et al., "Changes in EEG and autonomic nervous activity during meditation and their association with personality traits," International Journal of Psychophysiology, vol. 55, no. 2, pp. 199-207, 2005.

[10] A. A. Fingelkurts, A. A. Fingelkurts, and T. Kallio-Tamminen, "EEG-guided meditation: a personalized approach," Journal of Physiology-Paris, 2015.

[11] N. Singh and S. Telles, "Neurophysiological effects of meditation based on evoked and event related potential recordings,"
BioMed Research International, vol. 2015, Article ID 406261, 11 pages, 2015.

[12] A. Sobolewski, E. Holt, E. Kublik, and A. Wróbel, "Impact of meditation on emotional processing - a visual ERP Study," Neuroscience Research, vol. 71, no. 1, pp. 44-48, 2011.

[13] K.-M. Chang and P.-C. Lo, "F-VEP and alpha-suppressed EEGphysiological evidence of inner-light perception during Zen meditation," Biomedical Engineering: Applications, Basis and Communications, vol. 18, no. 1, pp. 1-7, 2006.

[14] H.-Y. Huang and P.-C. Lo, "EEG dynamics of experienced Zen meditation practitioners probed by complexity index and spectral measure," Journal of Medical Engineering and Technology, vol. 33, no. 4, pp. 314-321, 2009.

[15] K. Inanaga, "Frontal midline theta rhythm and mental activity," Psychiatry and Clinical Neurosciences, vol. 52, no. 6, pp. 555-566, 1998.

[16] Y. Kubota, W. Sato, M. Toichi et al., "Frontal midline theta rhythm is correlated with cardiac autonomic activities during the performance of an attention demanding meditation procedure," Cognitive Brain Research, vol. 11, no. 2, pp. 281-287, 2001.

[17] P.-C. Lo and Q. Zhu, "Microstate analysis of alpha-event brain topography during chan meditation," in Proceedings of the 8th International Conference on Machine Learning and Cybernetics, vol. 2, pp. 717-721, IEEE, Baoding, China, July 2009.

[18] P.-C. Lo and C.-H. Chang, "Spatially nonlinear interdependence of alpha-oscillatory neural networks under chan meditation," Evidence-Based Complementary and Alternative Medicine, vol. 2013, Article ID 360371, 12 pages, 2013.

[19] L. Zylowska, D. L. Ackerman, M. H. Yang et al., "Mindfulness meditation training in adults and adolescents with ADHD: a feasibility study," Journal of Attention Disorders, vol. 11, no. 6, pp. 737-746, 2008.

[20] D. A. Gusnard, E. Akbudak, G. L. Shulman, and M. E. Raichle, "Medial prefrontal cortex and self-referential mental activity: relation to a default mode of brain function," Proceedings of the National Academy of Sciences of the United States of America, vol. 98, no. 7, pp. 4259-4264, 2001.

[21] P. Qin and G. Northoff, "How is our self related to midline regions and the default-mode network?" NeuroImage, vol. 57, no. 3, pp. 1221-1233, 2011.

[22] A. A. Fingelkurts, A. A. Fingelkurts, S. Bagnato, C. Boccagni, and G. Galardi, "DMN operational synchrony relates to selfconsciousness: evidence from patients in vegetative and minimally conscious states," The Open Neuroimaging Journal, vol. 6, pp. 55-68, 2012.

[23] A. Berkovich-Ohana, J. Glicksohn, and A. Goldstein, "Mindfulness-induced changes in gamma band activity-implications for the default mode network, self-reference and attention," Clinical Neurophysiology, vol. 123, no. 4, pp. 700-710, 2012.

[24] B. R. Cahn, A. Delorme, and J. Polich, "Occipital gamma activation during vipassana meditation," Cognitive Processing, vol. 11, no. 1, pp. 39-56, 2010.

[25] A. Ahani, H. Wahbeh, H. Nezamfar, M. Miller, D. Erdogmus, and B. Oken, "Quantitative change of EEG and respiration signals during mindfulness meditation," Journal of NeuroEngineering and Rehabilitation, vol. 11, no. 1, article 87, 2014.

[26] S.-W. Xue, Y.-Y. Tang, R. Tang, and M. I. Posner, "Short-term meditation induces changes in brain resting EEG theta networks," Brain and Cognition, vol. 87, no. 1, pp. 1-6, 2014.

[27] A. S. Chan, Y. M. Y. Han, and M.-C. Cheung, "Electroencephalographic (EEG) measurements of mindfulness-based 
triarchic body-pathway relaxation technique: a pilot study," Applied Psychophysiology Biofeedback, vol. 33, no. 1, pp. 39-47, 2008.

[28] S. Warrenburg, R. R. Pagano, M. Woods, and M. Hlastala, "A comparison of somatic relaxation and EEG activity in classical progressive relaxation and transcendental meditation," Journal of Behavioral Medicine, vol. 3, no. 1, pp. 73-93, 1980.

[29] R. R. Pagano and S. Warrenburg, "Meditation: in search of a unique effect," in Consciousness and Self-Regulation, vol. 3, pp. 153-210, Plenum Press, New York, NY, USA, 1st edition, 1983.

[30] F. Travis, "Comparison of coherence, amplitude, and eLORETA patterns during transcendental meditation and TM-Sidhi practice," International Journal of Psychophysiology, vol. 81, no. 3, pp. 198-202, 2011.

[31] S. J. Grosswald, W. R. Stixrud, F. Travis, and M. A. Bateh, "Use of the transcendental meditation technique to reduce symptoms of attention deficit hyperactivity disorder (ADHD) by reducing stress and anxiety: an exploratory study," Current Issues in Education, vol. 10, no. 2, pp. 1-12, 2008.

[32] T. Krisanaprakornkit, C. Ngamjarus, C. Witoonchart, and N. Piyavhatkul, "Meditation therapies for attention-deficit/hyperactivity disorder (ADHD)," Database of Systematic Reviews, vol. 16, no. 6, 2010.

[33] D. A. Newande and S. S. Reisman, "Measurement of the electroencephalogram (EEG) coherence in group meditation," in Proceedings of the 22nd IEEE Annual Northeast Bioengineering Conference, pp. 95-96, IEEE, New Brunswick, NJ, USA, March 1996.

[34] R. Hebert, D. Lehmann, G. Tan, F. Travis, and A. Arenander, "Enhanced EEG alpha time-domain phase synchrony during transcendental meditation: implications for cortical integration theory," Signal Processing, vol. 85, no. 11, pp. 2213-2232, 2005.

[35] P. Eskandari and A. Erfanian, "Improving the performance of brain-computer interface through meditation practicing," in Proceedings of the 30th Annual International Conference of the IEEE Engineering in Medicine and Biology Society (EMBS '08), pp. 662-665, IEEE, Vancouver, Canada, August 2008.

[36] T. W. Kjaer, C. Bertelsen, P. Piccini, D. Brooks, J. Alving, and H. C. Lou, "Increased dopamine tone during meditation-induced change of consciousness," Cognitive Brain Research, vol. 13, no. 2, pp. 255-259, 2002.

[37] L. I. Aftanas and S. A. Golocheikine, "Non-linear dynamic complexity of the human EEG during meditation," Neuroscience Letters, vol. 330, no. 2, pp. 143-146, 2002.

[38] L. I. Aftanas and S. A. Golocheikine, "Human anterior and frontal midline theta and lower alpha reflect emotionally positive state and internalized attention: high-resolution EEG investigation of meditation," Neuroscience Letters, vol. 310, no. 1, pp. 57-60, 2001.

[39] S. Baijal and N. Srinivasan, "Theta activity and Meditative states: spectral changes during concentrative meditation," Cognitive Processing, vol. 11, no. 1, pp. 31-38, 2010.

[40] P. Arambula, E. Peper, M. Kawakami, and K. H. Gibney, "The physiological correlates of Kundalini Yoga meditation: a study of a Yoga master," Applied Psychophysiology Biofeedback, vol. 26, no. 2, pp. 147-153, 2001.

[41] R. Scheeringa, M. C. M. Bastiaansen, K. M. Petersson, R. Oostenveld, D. G. Norris, and P. Hagoort, "Frontal theta EEG activity correlates negatively with the default mode network in resting state," International Journal of Psychophysiology, vol. 67, no. 3, pp. 242-251, 2008.
[42] D. N. Ghista, D. Nandagopal, B. Ramamurthi, A. Das, A. Mukherju, and T. M. Krinivasan, "Physiological characterisation of the 'meditative state' during intuitional practice (the Ananda Marga system of meditation) and its therapeutic value," Medical and Biological Engineering, vol. 14, no. 2, pp. 209-214, 1976.

[43] K. C. Khare and S. K. Nigam, "A study of electroencephalogram in meditators," Indian Journal of Physiology and Pharmacology, vol. 44, no. 2, pp. 173-178, 2000.

[44] B. D. Elson, P. Hauri, and D. Cunis, "Physiological changes in yoga meditation," Psychophysiology, vol. 14, no. 1, pp. 52-57, 1977.

[45] Y.-J. Park and Y.-B. Park, "Clinical utility of paced breathing as a concentration meditation practice," Complementary Therapies in Medicine, vol. 20, no. 6, pp. 393-399, 2012.

[46] J.-F. Tsai, S.-H. Jou, W. C. Cho, and C.-M. Lin, "Electroencephalography when meditation advances: a case-based time-series analysis," Cognitive Processing, vol. 14, no. 4, pp. 371-376, 2013.

[47] D. Lehmann, P. L. Faber, S. Tei, R. D. Pascual-Marqui, P. Milz, and K. Kochi, "Reduced functional connectivity between cortical sources in five meditation traditions detected with lagged coherence using EEG tomography," NeuroImage, vol. 60, no. 2, pp. 1574-1586, 2012.

[48] A. A. Fingelkurts, A. A. Fingelkurts, and S. Kähkönen, "Functional connectivity in the brain-is it an elusive concept?" Neuroscience and Biobehavioral Reviews, vol. 28, no. 8, pp. 827836, 2005.

[49] T. Barnhofer, T. Chittka, H. Nightingale, C. Visser, and C. Crane, "State tate effects of two forms of meditation on prefrontal EEG asymmetry in previously depressed individuals," Mindfulness, vol. 1, no. 1, pp. 21-27, 2010.

[50] C. M. Goldstein, R. Josephson, S. Xie, and J. W. Hughes, "Current perspectives on the use of meditation to reduce blood pressure," International Journal of Hypertension, vol. 2012, Article ID 578397, 11 pages, 2012.

[51] H. Jaseja, "Potential role of self-induced EEG fast oscillations in predisposition to seizures in meditators," Epilepsy and Behavior, vol. 17, no. 1, pp. 124-125, 2010.

[52] A. Lutz, L. L. Greischar, N. B. Rawlings, M. Ricard, and R. J. Davidson, "Long-term meditators self-induce high-amplitude gamma synchrony during mental practice," Proceedings of the National Academy of Sciences of the United States of America, vol. 101, no. 46, pp. 16369-16373, 2004.

[53] A. V. Medvedev, A. M. Murro, and K. J. Meador, "Abnormal interictal $\gamma$ activity may manifest a seizure onset zone in temporal lobe epilepsy," International Journal of Neural Systems, vol. 21, no. 2, pp. 103-114, 2011.

[54] K. Kobayashi, M. Oka, T. Akiyama et al., "Very fast rhythmic activity on scalp EEG associated with epileptic spasms," Epilepsia, vol. 45, no. 5, pp. 488-496, 2004.

[55] M. A. Persinger, "Transcendental Meditation and general meditation are associated with enhanced complex partial epilepticlike signs: evidence for 'cognitive' kindling?" Perceptual and Motor Skills, vol. 76, no. 1, pp. 80-82, 1993.

[56] E. K. St. Louis and E. P. Lansky, "Meditation and epilepsy: a still hung injury," Medical Hypotheses, vol. 67, no. 2, pp. 247-250, 2006.

[57] S. R. Donaldson and P. B. Fenwick, "Effects of meditation," The American Journal of Psychiatry, vol. 139, no. 3, pp. 1217-1218, 1982.

[58] F. B. Vialatte, H. Bakardjian, R. Prasad, and A. Cichocki, "EEG paroxysmal gamma waves during bhramari pranayama: a yoga 
breathing technique," Consciousness and Cognition, vol. 18, no. 4, pp. 977-988, 2009.

[59] E. P. Lansky and E. K. St. Louis, "Transcendental meditation: a double-edged sword in epilepsy?" Epilepsy \& Behavior, vol. 9, no. 3, pp. 394-400, 2006.

[60] A. A. Fingelkurts and A. A. Fingelkurts, "EEG phenomenology and multiple faces of short-term EEG spectral pattern," The Open Neuroimaging Journal, vol. 4, pp. 111-113, 2010.

[61] A. A. Fingelkurts and A. A. Fingelkurts, "Brain-mind operational architectonics imaging: technical and methodological aspects," The Open Neuroimaging Journal, vol. 2, pp. 73-93, 2008.

[62] A. Gevins, "Electrophysiological imaging of brain function," in Brain Mapping. The Methods, A. W. Toga and J. C. Mazzoitta, Eds., pp. 175-188, Elsevier, New York, NY, USA, 2nd edition, 2002.

[63] S. Sanei and J. A. Chambers, EEG Signal Processing, John Wiley \& Sons, 2007.

[64] P. L. Nunez, "Physiological foundations of quantitative EEG analysis," in Quantitative EEG Analysis Methods and Clinical Applications, S. Tong and N. V. Thakor, Eds., pp. 1-22, Artech House, 2009.

[65] A. A. Fingelkurts and A. A. Fingelkurts, "Short-term EEG spectral pattern as a single event in EEG phenomenology," The Open Neuroimaging Journal, vol. 4, pp. 130-156, 2010.

[66] M. Teplan, "Fundamentals of EEG measurement," Measurement Science Review, vol. 2, no. 2, pp. 1-11, 2002.

[67] A. A. Fingelkurts and A. A. Fingelkurts, "Operational architectonics methodology for EEG analysis: theory and results," in Modern Electroencephalographic Assessment Techniques, vol. 91 of Neuromethods, pp. 1-59, Springer, New York, NY, USA, 2015.

[68] A. S. Chan, M.-C. Cheung, S. L. Sze, W. W.-M. Leung, and D. Shi, "Shaolin Dan Tian breathing Fosters relaxed and attentive mind: a randomized controlled neuro-electrophysiological study," Evidence-Based Complementary and Alternative Medicine, vol. 2011, Article ID 180704, 11 pages, 2011.

[69] E. Niedermeyer and F. H. Lopes da Silva, Electroencephalography: Basic Principles, Clinical Applications, and Related Fields, Lippincott Williams \& Wilkins, Philadelphia, Pa, USA, 2005.

[70] D. P. Subha, P. K. Joseph, R. Acharya U, and C. M. Lim, "EEG signal analysis: a survey," Journal of Medical Systems, vol. 34, no. 2, pp. 195-212, 2010.

[71] C. S. Herrmann, T. Arnold, A. Visbeck, H.-P. Hundemer, and H. C. Hopf, "Adaptive frequency decomposition of EEG with subsequent expert system analysis," Computers in Biology and Medicine, vol. 31, no. 6, pp. 407-427, 2001.

[72] H. Sharabaty, J. Martin, B. Jammes, and D. Esteve, "Alpha and theta wave localisation using hilbert-huang transform: empirical study of the accuracy," in Proceedings of the 2nd International Conference on Information \& Communication Technologies, pp. 1159-1164, Damascus, Syria, 2006.

[73] P.-C. Lo and J.-S. Leu, "Quantification of pseudo-periodicity of alpha rhythm in meditation EEG," Journal of Medical and Biological Engineering, vol. 25, no. 1, pp. 7-13, 2005.

[74] B. S. Raghavendra and D. N. Dutt, "Multiscale fractal dimension technique for characterization and analysis of biomedical signals," in Proceedings of the Digital Signal Processing and Signal Processing Education Meeting (DSP/SPE '11), pp. 370-374, IEEE, Sedona, Ariz, USA, January 2011.

[75] G. Nolfe, "EEG and meditation," Clinical Neurophysiology, vol. 123, no. 4, pp. 631-632, 2012.
[76] C. M. Michel and M. M. Murray, "Towards the utilization of EEG as a brain imaging tool," NeuroImage, vol. 61, no. 2, pp. 371385, 2012.

[77] E. M. Whitham, K. J. Pope, S. P. Fitzgibbon et al., "Scalp electrical recording during paralysis: quantitative evidence that EEG frequencies above $20 \mathrm{~Hz}$ are contaminated by EMG," Clinical Neurophysiology, vol. 118, no. 8, pp. 1877-1888, 2007.

[78] P. Kellaway, "An orderly approach to visual analysis: characteristics of the normal EEG of adults and children," in Current Practice of Clinical Electroencephalography, D. Daly and T. Pedley, Eds., Raven Press, New York, NY, USA, 2nd edition, 1990.

[79] C. M. Ryan and B. J. Murray, "An unexpected abnormality on the EEG," Journal of Clinical Sleep Medicine, vol. 6, no. 6, pp. 613-615, 2010.

[80] D. C. Hammond, "Compendium of common terms in EEG and neurofeedback," University of Utah School of Medicine, Board Certified in EEG, QEEG and Neurophysiology, EEG and Neurosciense Society.

[81] X. Wu and X. Q. Liu, "Study of the $\alpha$ frequency band of healthy adults in quantitative EEG," Clinical EEG Electroencephalography, vol. 26, no. 2, pp. 131-136, 1995. 


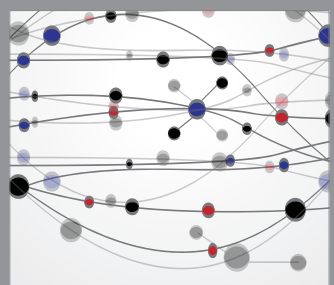

The Scientific World Journal
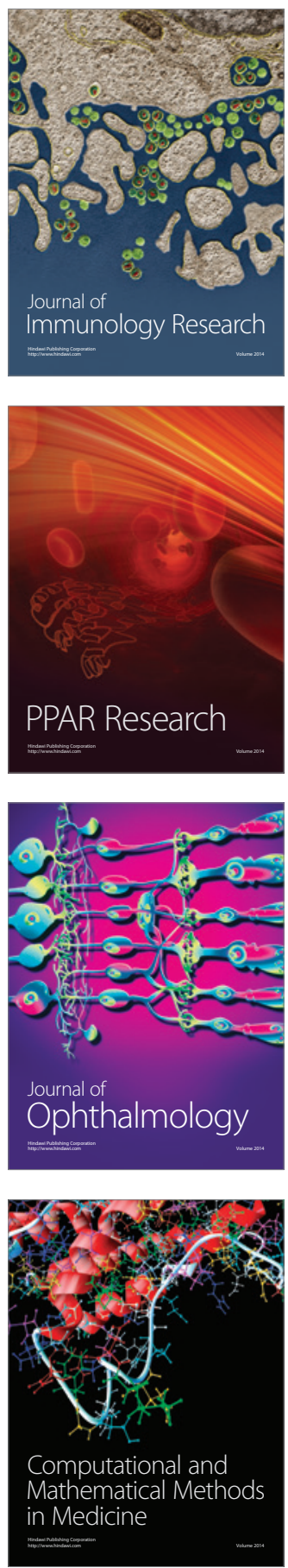

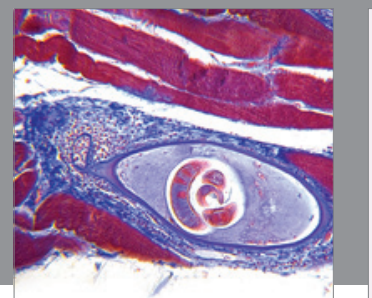

Gastroenterology

Research and Practice
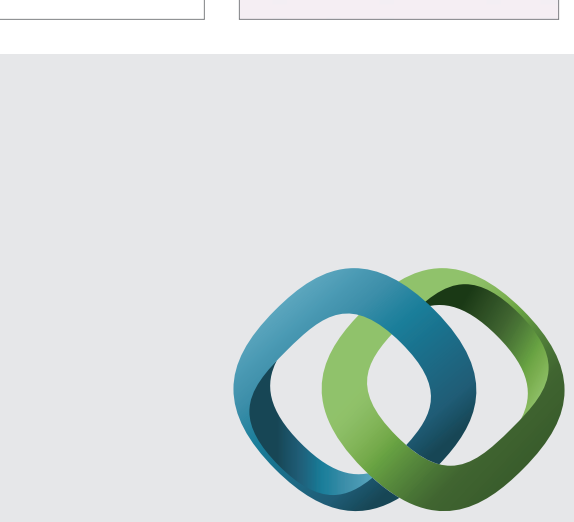

\section{Hindawi}

Submit your manuscripts at

http://www.hindawi.com
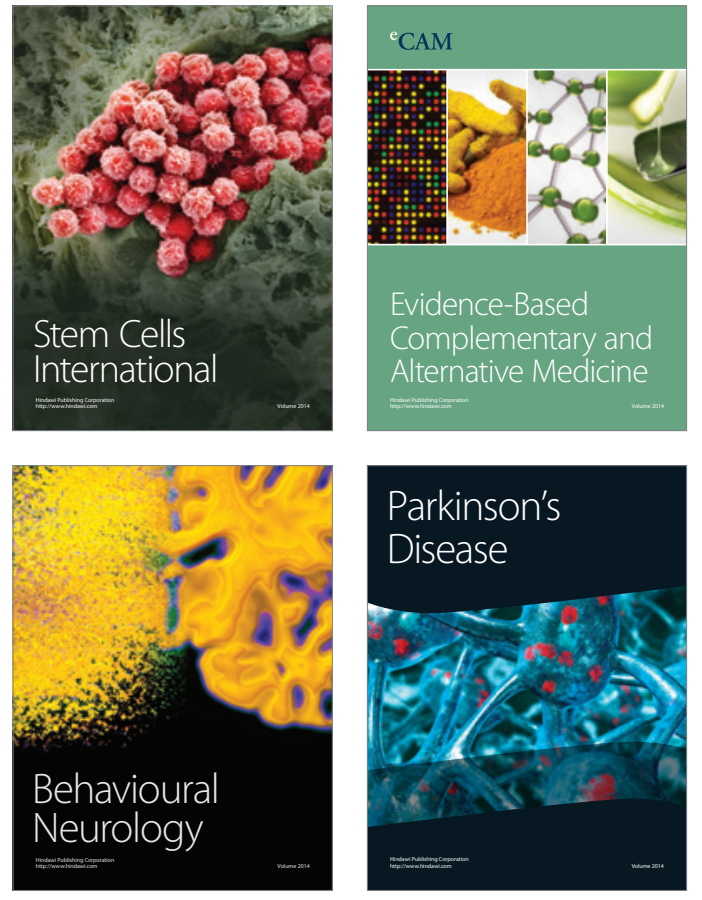
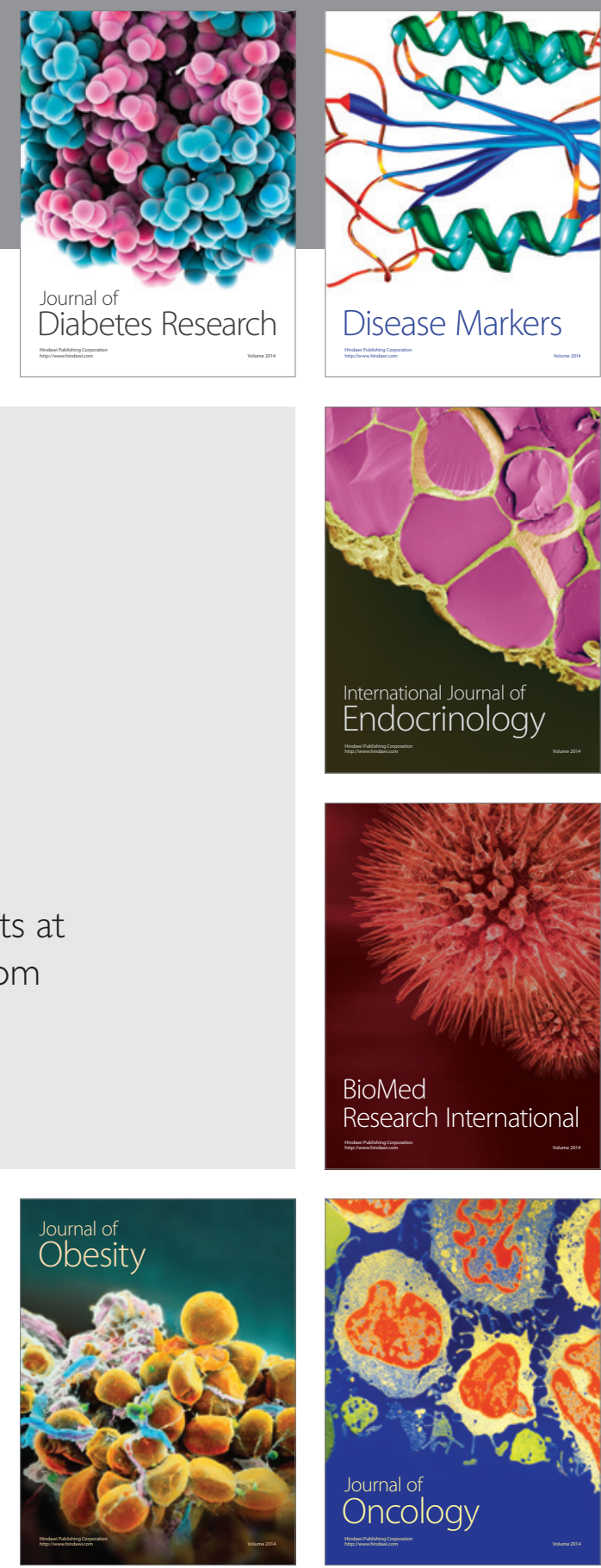

Disease Markers
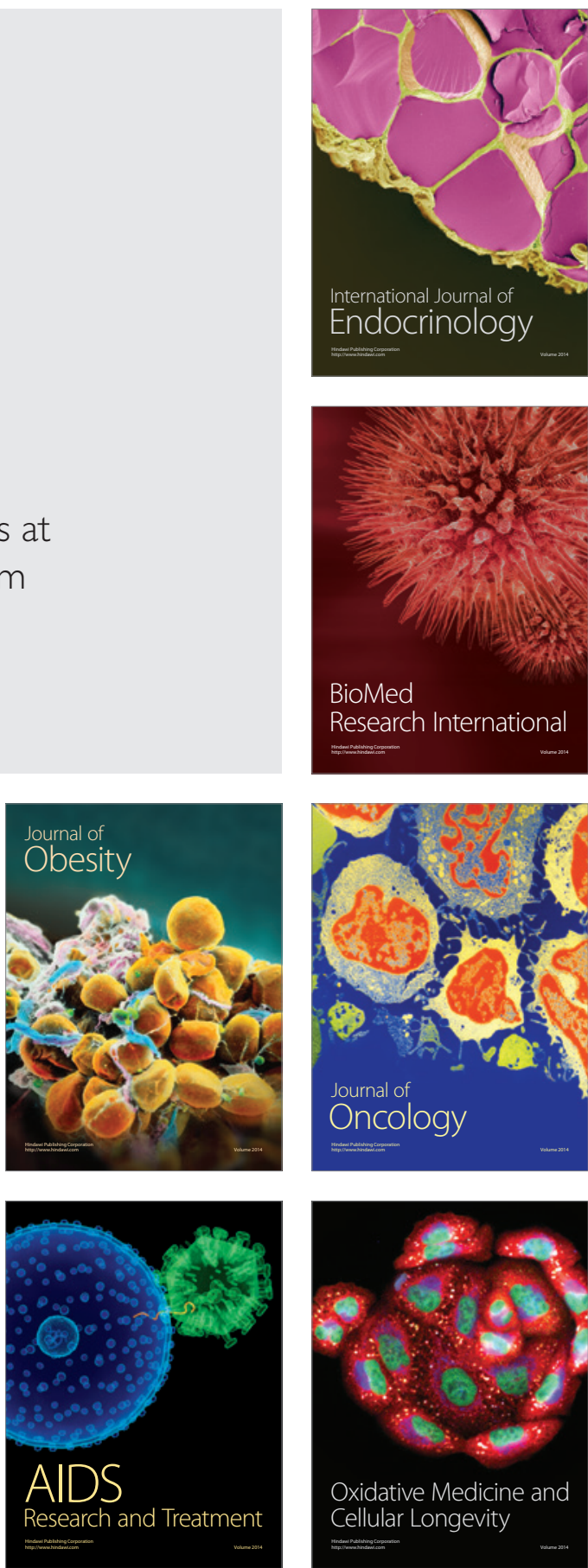